\title{
Research Method of Forecasting the Equipment Maintenance and ordnance Demand
}

\author{
Xiuhua Wang \\ Department of Graduate Management, Equipment \\ Academy, Beijing, China \\ wangxiuhuade1@163.com
}

\begin{abstract}
Equipment maintenance demand forecasting plays a very important role in equipment maintenance support work. Three ways of forecasting methods are displayed in this paper, they are equipment maintenance demand forecasting method based on historical data, reliability-based method and method based on artificial intelligence. There are some deficiencies exist in these three methods, so this paper also proposed these problems and analyzed their causes. Finally, suggestions on how to improve the scientificity and accuracy of forecasting models are made in this article.
\end{abstract}

Keywords- equipment maintenance, ordnance inventory, demand forecasting

\section{INTRODUCTION}

During equipment maintenance and ordnance inventory management activities, scientifically forecasting quantity of demand and reasonably determine inventory types and their amount has very important significance. When there is too little inventory, it is difficult to guarantee the operational readiness of equipment while excessive inventory will result in a waste of resources and has a bad impact on the real needs of the equipment maintenance ordnance inventory. To this end, both domestic and abroad scholars are attaching great importance to the research of equipment maintenance ordnance demand forecasting methods.

In the 1950s, RAND Corporation began to study equipment maintenance ordnance demand forecasting problems. In the early days of this research, the most representative achievement is Feeney and Sherbrooke's result. They not only developed the theory of demand forecasting equipment maintenance equipment, also combined it with inventory control problem, putting forward the multi-echelon inventory control technology which began to be used in the US air force in 1975 to maintain equipment.

Because Feeney and Sherbrooke's research has made great success, in the next ten years from then, there was no more influential research results about demand forecasting. Until the 1980s, the demand forecasting was again aroused the attention of researchers and shift from the usual demand model to wartimeequipment maintenance and ordnance support. The earliest research of this kind was made by Muckstadt in 1980 using unsteady Poisson distribution to describe the requirements process.

In China, the equipment maintenance equipment demand forecasting has gradually become one of the hottest research topics in recent years. The researchers developed a large number of demand forecasting methods, such as non-

\author{
Zhengping Shu \\ Department of Graduate Management, Equipment \\ Academy, Beijing, China \\ wangxiuhuade1@163.com
}

homogeneous Poisson process regression, gray theory, Bayesian theory, seasonal index and so on. But these prediction methods are based on individual equipment maintenance, which is on the basis of a single species of equipment maintenance ordnance consumption data to make predictions. However, the weaponry is composed of numerous equipment maintenances. The actual work of equipment maintenance is usually under a certain cost constraint, using the results of the prediction, to determine the quantity of each equipment maintenance andordnance inventory to make the whole equipment system has the highest availability. Predition method based on individual equipment maintenance, therefore, is not easy to security personnel to use when making support decisions. Therefore, equipment maintenance ordnance forecasting method based on systematic equipment became a focus of the study

\section{FORECASTING METHODS}

Forecasting equipment and ordnance maintenance is a very important link in equipment maintenance management work. A great number of scholars from different perspectives forecasted demand for equipment maintenance, according to the principle of prediction methods, the existing equipment maintenance demand forecasting methods can be divided into the following three ways: method based on historical data, method based on reliability and the method based on artificial intelligence and so on.

\section{A. Equipment maintenance demand forecasting method based on historical data}

Forecasting method based on historical data mainly analyze the historical data, then find out the law of equipment maintenance consumption law, to establish an appropriate forecasting model, predicting future demand for equipment maintenance. Such methods include Exponential Smoothing, Croston method, Bootstrap method, fitting distribution method as well as their improving methods.

Limitation: This method requires a high accuracy for historical data, while due to the structure of the equipment maintenance work and some other reasons, it is really hard to preserve some important data completely, so we can only use some past experience data, which led to the decline in data accuracy, making a lot differences between the prediction results and the actual outcomes. 


\section{B. Equipment maintenance demand forecasting method based on reality}

Reality-based approach is mainly rely on the reliability of the components itself, according to the probability of occurrence of equipment failure in different working lengths to predict the equipment maintenance consumption over a period of time in the future.

Limitation: Reliability prediction methods are usually based on the life distribution assumption for components, which often is very strict, and therefore it is subjected to many limitations in practical application.

\section{Equipment maintenance demand forecasting method based on artificial intelligence}

This method mainly use artificial neural network or support vector machine and other artificial intelligence methods or tools to study the rule of equipment maintenance equipment demand.

\section{1) Artificial neural network (ANN)}

Artificial neural network prediction method is a hot research method currently, which is focus on human body or biological neural network abstraction and modeling. It is a kind of highly nonlinear systems with the ability to learn from the environment. Theoretically it can approach any nonlinear function which definition is intensive on arbitrary precision approximation. ANN mainly applies the neural network based machine learning method, by determining the network structure, build a predictive model to forecast future demand.

ANN is widely applied to demand forecasting problems now. With the aim of improving prediction accuracy, people use genetic algorithm, fuzzy system and expert knowledge system method to improve basic ANN constantly.

Limitation: Due to the slow convergence of neural network, poor ability of global search and lack of statistical significance, when the sample data is limited and there is noise in the system approach, it will inevitably fall into the problem of over learning or less leaning. Although data can be filtered before fitting and it can achieve a certain effect, but in the case of unknown noise probability density, its essence can only have an effect of average smoothing.

\section{2) Support vector machine (SVM)}

Support vector machine is the youngest and the most practical part of the statistical learning theory. It is not just a theoretical analysis tool, it is also a learning algorithm can be constructed to predict multidimensional forecasting tools, making abstract learning theory can be converted to common practical prediction algorithm. Because SVM method has the minimum structure risk, strong generalization ability, global optimal solution and many other advantages, id can solve the small sample learning problems and avoid over learning trouble and dimension disaster problem. These features are not as good as in other learning algorithms.

Limitation: Prediction method based on artificial intelligence algorithm requires users themselves have the full understanding of the characteristics. Various parameters of the algorithm need to be set and adjusted artificially with a high degree of subjectivity and randomness. And these algorithms require a lot of training and testing samples. All of these factors limit the application of the artificial intelligence method.

\section{EXISTING PROBLEMS}

At present, a lot of work has been done in the aspect of equipment maintenance ordnance demand forecasting both at home and abroad, which also obtained certain achievements, but some problems are still exist.

\section{A. There is a blind spot when studying the law of current equipment maintenance ordnance demand}

Due to the law of equipment maintenance and ordnance demand influenced by many areas such as maintenance mode, maintenance level, maintenance strategy and so on, so the problem of background difference produced a varity of equipment maintenance demand, many questions need for targeted modeling work. But the exiting research only satisfy a portion of the equipment maintenance ordnance management work, there are still many issues to be studied in depth.

For the first aspect, the existing research focuses on single-level security structure, there is no consideration of the impact of equipment maintenance and security level of demand for equipment maintenance. The maintenance and guarantee level will have a significant impact on equipment maintenance demand rule, for example, at the grassroots level and intermediate level maintenance process, due to the repair practices, equipment maintenance and equipment replacement will lead to different standards of maintenance needs of law equipment maintenance equipment changes. Therefore, according to the multi-level structure of the structure in our country's military equipment, researches on laws of equipment maintenance demand for each level should be carried out.

Another improvement is about the study of the law of equipment maintenance demand under different maintenance strategies. There will have a direct impact on equipment maintenance when maintenance strategy changes, while the existing model study the part of the maintenance strategy, there are still a lot of maintenance strategies widely applied in the practical work lack the corresponding equipment maintenance and ordnance requirement model, such as using the maintenance strategy defines the maximum number of components and complex system using timed maintenance and regular replacement combined strategy all need a further study.

The last one is due to the restriction of modeling tools, the existing equipment maintenance demand model mainly focus on single component system, less consider the complex structure of equipment. And limited demand rule of equipment maintenance on multi-component system for the distribution of the various components, such as life distribution, there also has strict requirement, resulting in a narrow application of models. 


\section{B. Poor versatility for existing analytical model of equipment maintenance inventory}

In order to facilitate analysis and processing, the existing analytical model in equipment inventory maintenance usually simplified assumptions, generally choose the poisson process and update some process such as described the arrival situation of equipment maintenance demand, while make ordering time and maintenance time certain value or follow the typical distribution such exponential distribution. This makes the model can only apply to some specific issues, when minor changes made in assumptions, the existing model method and the conclusion will no longer applicable, which meansversatility of the model is poor.

\section{REASON ANALYSIS}

Because of the complexity of equipmentmaintenance system, randomness of equipment failure and specificity of equipment consumption, there is no doubt that it will lead to increase the difficulty in demand analyzing and forecasting. The reasons why it is so difficult to forecast the equipment demand are as follows.

Firstly, the needs of equipmentoccurred discontinuity, there are large zero value in data. In addition, the discontinuity demands are not simply equal to continuous demands, although this transfer simplified the forecast method, the model has lost some important characteristics of descriptive object. For example, equipment demand is low usually and time lasts long between two needs. If there is a big difference between continuous demand which is measured in the longer period of time and reality problem, therefore, the forecast would become meaningless.

Secondly, there are many reasons to cause the demand of equipment. In addition to regular equipment reliability, the way of equipment use, maintenance and repair strategies all lead to affect the occurrence time and number of equipment demand. In the adjacent time, with equipment use and aging, equipment would show a certain self correlation. This indicates there is a certain relationship between demand of equipment and equipment maintenance, use of equipment. Because related to these complex relationships which makes the demand of equipment had great fluctuation and it shows significant "burstiness" and "jumping”.

Thirdly, the historical data of equipment maintenance data is limited, so the prediction method is restricted.

\section{COUNTERMEASURES AND SUGGESTIONS}

Currently, our army equipment maintenance demand is not timely and accurate, which can not meet the needs of equipment support under the condition of information, even can not meet the low intensity training equipment demand in peacetime. To overcome the equipment maintenance ordnance demand plan and inventory size problem, we should strengthen research of equipment maintenance ordnance demand forecasting model, improve the ability of equipment maintenance ordnance demand forecast and inventory calculation.

\section{A. Strengthen equipment maintenance and ordnance demand forecasting methods and its theoretical research model}

Theoretical research is a guide of empirical study, only in-depth study of the theory in detail of equipment maintenance ordnance demand forecast theoretically, can we accurately grasp the rule and method of equipment maintenance material demand forecast, grasp the domestic and foreign study the degree of equipment maintenance ordnance demand forecasting and the development movement in this area, understand its trend to carry out targeted research. Take US army as example, every year they organize inner military research institution and pirate universities to carry out equipment maintenance requirement and the simulation modeling research. Thus, once the US army's combat mission or equipment system changed significantly, they can immediately eliminate old algorithm and models and put a new applicable calculation procedure and model into use to adapt to the new situation and needs. Our army has also organized research institutions to carry out similar studies, but few achievements have been made and the study did not continuity. We also should make equipment maintenance demand forecasting method as a long-term and foundational work.

\section{B. Simplify the equipment maintenance demand forecast method combined with out actual situation}

In actual equipment maintenance management work, due to the security personnel skill levels, working accuracy, timeliness and other various restrictive factors, algorithm and the model used to predict equipment maintenance demand maybe not very complex or elaborately. To judge whether an algorithm or a model good, the key is to see whether it can meet the actual needs of troops under acceptable accuracy with the simplest operation, the lowest cost and the shortest time. In abroad, algorithms and models used in real life are relatively simple, a high school education level soldier can master them normally after a simple training. For our army, we should based on algorithms and models we already have, combined with the actual needs of the troops and the extent of the skills of out military soldiers and officers, to optimize these developed theoretical algorithms and models through engineering approaches. Only the algorithms and forecasting models match with our maintenance decision-making level, can we promote scientific equipment maintenance demand forecasting and inventory management.

\section{Employ unified technical standards for equipment maintenance demand forecasting model}

Standardization is the trend of future development of the model. The US army equipment maintenance supply model basically adopted a High Level Architecture (HLA) standard for interconnection between models, which laid the foundation for models to communicate and integrate with each other. We must pay great attention and take advantage of this trend in modeling equipment maintenance demand forecasting methods. During the process of model development, other armed services personnel from other 
institutions should also be absorbed, for the subsequent implementation of unified army equipment maintenance. To achieve this goal, the headquarters should give policy support and issue relevant policy documents, for the realization of the standardization of equipment maintenance demand forecasting methods, policy guidance and long-term development plan are also in need, in addition, specialized consultants must be set up to give specific guidance.

\section{REFERENCES}

[1] CharytoniukW.,Box E.D., Lee W.J.,etal.Neuralnetwork-based demandforecasting in a deregulated environment [J]. IEEE Transactions on IndustryApplications, 2000. 36(3): 893-898.
[2] Gorucu F.B., Geris P.U., Gumrah F. Artificial neural network modelingforforecasting gas consumption [J]. Energy Sources, 2004. 26(3): 299-307.

[3] Wang N.C.,KangR.,Research of equipment demand generation, transmission and parsing algorithm.[J].Journal of aviation, 2008. 29(5):1163-1167.

[4] Kennedy W.J., Patterson J.W., Fredendall L.D. An overview ofrecentliteratureon spare parts inventories [J]. International Journal of Production Economics,2002.76(2):201-215.

[5] Willemain T.R., Smart C.N., Schwarz H.F. A new approach to forecastingintermittent demand for service parts inventories [J]. International Journal ofForecasting, 2004. 20(3): 375-387. 